Results from (5), (7), (8) and (9) can be sent to a printer.

\title{
15 Can I easily crash STATCALC?
}

All the programs check that you do not make obvious mistakes. For example, when entering the data, if you type the letter I instead of the digit 1 you will get an error message and a bleep to attract your attention. Other checks such as invalid option requests, invalid transformations and selecting the printer when it is not switched on, are all captured.

However, no attempt has been made to capture every error you may make because it would severely limit the memory space available for your data.

\section{How can I transform my data?}

FILER, UNIVAR, BIVAR and POLYVAR allow you to transform or convert your measurements. For example, you may have a variable measured in miles which you wish to convert to kilometres or you may have a variable measured in degrees Fahrenheit which you wish to convert to degrees Centigrade. Where you have bivariate or multivariate data you can apply different transformations to each variable. The programs will provide you with specific details of how to transform your data but in general terms any valid BBC BASIC formula is acceptable as a transformation provided that it does not generate computer errors, such as dividing by zero or determining the logarithm of zero. 